\title{
Positron annihilation rate in single atom with slater type orbital approximation
}

\author{
- Trinh Hoa Lang \\ - Chau Van Tao \\ - Le Hoang Chien \\ - Huynh Thi Yen Hong \\ - Huynh Ngoc Tram \\ University of Science, VNU-HCM \\ (Received on March 20 $0^{\text {th }} 2013$, accepted on March 28 ${ }^{\text {th }}$ 2014)
}

electrons and positron wave functions. The kinetic energies of the electrons and positron were treated on the same footing, and the Born-Oppenheimer approximation was applied to the nuclei. In this paper we treated only those systems for the valance electrons in the real spatial coordinate of the atom or molecule. The complex of many-particle problem was solved by the Schrongdinger of one particle equation which is derived by Kohn-Sham approximation and single particle wave function of Slater type orbital. As a result of this model, the positron annihilation rate and lifetime in some atoms, $\mathrm{Ti}, \mathrm{Zn}$ and $\mathrm{Zr}$, were calculated. positron and electron electron correlation in Keywords: Positron, annihilation, correlation, positron, VQMC.

\section{INTRODUCTION}

The structure of material is studied by positron lifetime spectrum, which has many lifetime components depending on a local structure where a positron annihilates with electrons. The problem is how to determine accurately lifetime components of positron from experiments. It pursues to encourage studying deeply the positron annihilation in element specific structure of materials such as single atom, molecule or unit cell.
In this work, the study of positron annihilation with valence electrons of unit structure of material is assumed that positron will bind with this unit to form new state through these valence electrons before it destroys with one of these. This bound state of positron and this unit must be in ground state and then in this ground-state positron will destroy with electron. By this assumption, the orthonormalized Slater type orbital will be used for describing electrons 
and positron wave functions in the irreducible element and VQMC [8] will be used to find the ground-state wave function for electrons and positron.

When a positron is going into the material, positron will be slowed down to thermal energy and then bound with atoms in the unit structure before annihilate with valence electrons of these atoms. In this state, positron wave function is approximated by hybrid wave function of valence electrons.

To find ground state of this system, it is reduced the complicated many-body problem that the energy of each particle is minimized rather than total energy. The total wave function, Slater determinant which represented for exchange effect, is avoided by solving individual particle equation with Hamiltonian of exchange potential. One-particle energy will be derived by one particle equation, which is constructed from Kohn - Sham method and single particle wave function. This wave function consists of atomic wave function and correlation function. By ideal of choosing trial single wave function, variational quantum Monte Carlo method is used to find the ground state of positron - valence electron of unit element.

In this scenario, the optimized electron and positron distributions in real space of unit structure of material is determined by the minimal energy. From this distribution, the other quantities will be calculated by Monte Carlo method.

\section{MATERIALS AND METHOD THEORY}

To study the positron annihilation in the unit structure of material, the two - component density - functional theory [3] is used with some modification in single particle wave function and exchange - correlation potential. A singleelectron wave function now is not only pure atomic wave function but also included the correlation function of electron - electron and electron - positron. Therefore, the correlation effects are contained in this correlation function. The set of one-particle Schrodinger equations for electrons and positrons is given by

$-\frac{1}{2} \nabla^{2} \Psi_{\mathrm{i}}(\mathrm{r})+\left[\mathrm{V}_{\mathrm{x}}\left(\rho^{-}\right)-\phi(\mathrm{r})\right] \Psi_{\mathrm{i}}(\mathrm{r})=\varepsilon_{\mathrm{i}} \Psi_{\mathrm{i}}(\mathrm{r})$

$-\frac{1}{2} \nabla^{2} \Psi_{\mathrm{i}}^{+}(\mathrm{r})+\left[\mathrm{V}_{\mathrm{x}}\left(\rho^{+}\right)+\phi(\mathrm{r})\right] \Psi_{\mathrm{i}}^{+}(\mathrm{r})=\varepsilon_{\mathrm{i}}^{+} \Psi_{\mathrm{i}}^{+}(\mathrm{r})$

with

$$
\Psi_{\mathrm{i}}(\mathrm{r})=\varphi_{\mathrm{i}}(\mathrm{r}) \mathrm{J}(\mathrm{r})
$$

where $\varphi_{\mathrm{i}}$ is atomic wave function for single electron or positron wave function; $J(r)$ is the correlation function of electron - electron or electron - positron. These functions are parameterize functions. The electron and positron densities are calculated by summing over the occupied states ( $n_{-}$and $n_{+}$are the number of electrons and positrons respectively).

$$
\rho^{-}=\sum_{\mathrm{i}}^{\mathrm{n}_{-}}\left|\Psi_{\mathrm{i}}(\mathrm{r})\right|^{2} ; \rho^{+}=\sum_{\mathrm{i}}^{\mathrm{n}_{+}}\left|\Psi_{\mathrm{i}}^{+}(\mathrm{r})\right|^{2}
$$

The Slater type wave functions are used for single electron wave function and linear combination of these is used for single positron wave function. The correlation function has form $\mathrm{J}(\mathrm{r})=\mathrm{e}^{-\mathrm{u}(\mathrm{r})}$ and $\mathrm{u}(\mathrm{r})$ is Jastrow function. This function is suggested in the new modified Jastrow function which is a combination of Padé [8], long correlation, and Yukawa [5], short correlation. The Jastrow function for electron electron correlation is given by

$$
u^{e e}(r)=\frac{\beta_{e}}{1+\alpha_{e} r}+\frac{A_{e}\left(1-e^{-F_{e} r}\right)}{r}
$$

and for electron - positron correlation is given by

$$
u^{e p}(r)=\frac{\beta_{e p} r}{1+\alpha_{e p} r}+\frac{A_{e p}\left(1-e^{-F_{e p} r}\right)}{r}
$$

where $\beta_{\mathrm{e}}, \alpha_{\mathrm{e}}, \mathrm{A}_{\mathrm{e}}, \mathrm{F}_{\mathrm{e}}, \beta_{\mathrm{ep}}, \alpha_{\mathrm{ep}}, \mathrm{A}_{\mathrm{ep}}$, and $\mathrm{F}_{\mathrm{ep}}$ are the variational parameters. These parameters depend on the electronic structure of material.

\section{Trang 44}


If the exchange - correlation potential is separated into exchange and correlation potential, then the correlation potential is hidden in the correlation wave function, and the exchange potential for a uniform degenerate electron gas is adapted for use in atomic calculation by using local electron density as given by [4]

$$
V_{x}(\rho)=\varepsilon_{x}+\frac{d \varepsilon_{x}}{d \rho}
$$

where $\varepsilon_{x}$ is exchange energy per electron of a uniform electron gas with density $\rho$.

$$
\varepsilon_{\mathrm{x}}=-\frac{3}{4}\left(\frac{3}{\pi} \rho\right)^{1 / 3}
$$

Now $\rho$ is replaced by local electron density in an atom.

The equations (1) and (2) are solved with the wave function in (3), (5) and (6) to find ground state of unit structure - positron system by VQMC, which minimize one electron and positron energy to obtain their ground state wave function.

The pair correlation function of electron and positron is built from data of Monte - Carlo simulation which is obtained from these ground states. The analytical form of pair correlation function is fitted in Chebyshev polynomial. Then the positron annihilation enhancement is determined by extrapolation of this function at distance between electron and positron which is zero.
Positron annihilation rate depending on the overlap of electron and positron densities and enhancement factor is directly given by the value of the pair-correlation function at the origin via the relation $[7,9]$

$$
\Gamma=\frac{\pi \mathrm{r}_{\mathrm{e}}^{2} \mathrm{c}}{\Omega} \sum_{\mathrm{i}}^{\mathrm{N}} \int \rho_{\mathrm{i}}^{-}(\mathrm{r}) \rho^{+}(\mathrm{r}) \mathrm{g}\left(0 ; \rho^{-}, \rho^{+}\right) \mathrm{dr}
$$

where $g\left(0 ; \rho^{-}, \rho^{+}\right)$is the enhancement factor which is the value of the pair - correlation function $\mathrm{g}\left(\mathrm{r} ; \rho^{-}, \rho^{+}\right)$at $\mathrm{r}=0$ for given electron density. This function is fitted in Chebyshev polynomial [11] as given by

$$
\mathrm{g}\left(\mathrm{r} ; \rho^{-}, \rho^{+}\right) \equiv \mathrm{g}(\mathrm{r})=\sum_{\mathrm{i}=0}^{\mathrm{N}} \mathrm{c}_{\mathrm{i}} \mathrm{T}_{\mathrm{i}}\left(\frac{2 \mathrm{r}-\mathrm{L}}{\mathrm{L}}\right)
$$

$\mathrm{L}$ is maximum interaction range of electron and positron, radius of spherical cell, which is used for collecting electron distribution data in space around a positron.

This model is applied to study the positron annihilation in the single atoms of titanium, zinc and zirconium. The single-particle wave function of these atoms are constructed by the Slater Type Orbital[6]. The wave function of the valance electrons of these atoms are in the $3 \mathrm{~d}, 4 \mathrm{~s}, 4 \mathrm{~d}$ and 5 s shells.

The single - particle wave function for the positron in the bound state with valance electrons of each atom is hybrid wave function of the valance electrons of this atom. According to the principle of linear superposition, the single particle wave function for positron in titanium is supposed to take the form as

$$
\begin{aligned}
\varphi_{\mathrm{i}}^{\mathrm{p}}\left(\mathrm{Zp}_{\mathrm{Ti}}, \mathrm{r}\right)= & \mathrm{c}_{1} \varphi_{\mathrm{i}}^{3 \mathrm{dz} 2}\left(\mathrm{Zp}_{\mathrm{Ti}}, \mathrm{r}\right)+\mathrm{c}_{2} \varphi_{\mathrm{i}}^{3 \mathrm{dxz}}\left(\mathrm{Zp}_{\mathrm{Ti}}, \mathrm{r}\right)+\mathrm{c}_{3} \varphi_{\mathrm{i}}^{3 \mathrm{dyz}}\left(\mathrm{Zp}_{\mathrm{Ti}}, \mathrm{r}\right) \\
& +\mathrm{c}_{4} \varphi_{\mathrm{i}}^{3 \mathrm{~d} x 2-\mathrm{y} 2}\left(\mathrm{Zp}_{\mathrm{Ti}}, \mathrm{r}\right)+\mathrm{c}_{5} \varphi_{\mathrm{i}}^{3 \mathrm{dxy}}\left(\mathrm{Zp}_{\mathrm{Ti}}, \mathrm{r}\right)+\mathrm{c}_{6} \varphi_{\mathrm{i}}^{4 \mathrm{~s}}\left(\mathrm{Zp}_{\mathrm{Ti}}, \mathrm{r}\right)
\end{aligned}
$$

where $c_{1}, c_{2}, c_{3}, c_{4}, c_{5}$ and $c_{6}$ are the weighted coefficients of positron wave function with $c_{1}=c_{2}=$ $c_{3}=c_{4}=c_{5}=c_{6}=1 / \sqrt{ } 6$.

The positron wave function in zinc is given by 


$$
\varphi_{\mathrm{i}}^{\mathrm{p}}\left(\mathrm{Zp}_{\mathrm{Zn}}, \mathrm{r}\right)=\sqrt{\frac{\mathrm{Zp}_{\mathrm{Zn}}^{3}}{333 \pi}}\left(4 \mathrm{Z} \mathrm{p}_{\mathrm{Zn}}^{3} \mathrm{r}^{3}-18 \mathrm{Zp}_{\mathrm{Zn}}^{2} \mathrm{r}^{2}+18 \mathrm{Z} \mathrm{p}_{\mathrm{Zn}} \mathrm{r}-3\right) \mathrm{e}^{-\mathrm{Zp}_{\mathrm{Zn}} \mathrm{r}}
$$

And the positron wave function in zirconium is given by

$$
\begin{aligned}
\varphi_{\mathrm{i}}^{\mathrm{p}}\left(\mathrm{Zp}_{\mathrm{Zr}}, \mathrm{r}\right)= & \mathrm{c}_{1} \varphi_{\mathrm{i}}^{4 \mathrm{dz} 2}\left(\mathrm{Zp}_{\mathrm{Zr}}, \mathrm{r}\right)+\mathrm{c}_{2} \varphi_{\mathrm{i}}^{4 \mathrm{dxz}}\left(\mathrm{Zp}_{\mathrm{Zr}}, \mathrm{r}\right)+\mathrm{c}_{3} \varphi_{\mathrm{i}}^{4 \mathrm{dyz}}\left(\mathrm{Zp}_{\mathrm{Zr}}, \mathrm{r}\right) \\
& +\mathrm{c}_{4} \varphi_{\mathrm{i}}^{4 \mathrm{~d} \times 2-\mathrm{y} 2}\left(\mathrm{Zp}_{\mathrm{Zr}}, \mathrm{r}\right)+\mathrm{c}_{5} \varphi_{\mathrm{i}}^{4 \mathrm{dxy}}\left(\mathrm{Zp}_{\mathrm{Zr}}, \mathrm{r}\right)+\mathrm{c}_{6} \varphi_{\mathrm{i}}^{5 \mathrm{~s}}\left(\mathrm{Zp}_{\mathrm{Zr}}, \mathrm{r}\right)
\end{aligned}
$$

Using equations from (1) to (8) and the electron and positron wave functions, the minimizing energy is calculated by VQMC and then ground-state wave function of the positrontitanium, zinc and zirconium systems can be obtained.

\section{RESULTS AND DISCUSSION}

In calculation of ground-state wave function of electrons and positron in titanium, zinc and zirconium, for each of these atoms, the ten sets of energy data which correspond to the variation of the ten parameters of electron and positron wave functions are generated. Each energy is calculated by varying one of these parameters, and the other nine parameters are kept as constants. The sets of optimized parameters, which make the trial wave function well approach to exact wave function, are determined from these sets by minimization of energy. These optimized parameters of titanium, zinc and zirconium are given in Table 1, Table 2 and Table 3. From these optimized parameter, the pair correlation distributions and annihilation rates are estimated.

\section{The results of the enhancement factor and positron lifetime in titanium}

The enhancement factor and positron lifetime in single atomic titanium are determined by using the parameters in Table 1. The electron positron pair correlation distribution of titanium is collected by Monte - Carlo's simulation as shown in Fig. 1a. The enhancement factor is extrapolated by pair correlation function, fitted by Chebyshev polynomial in equation (10).

Table 1. The values of optimized parameters of electron and positron wave function of titanium

\begin{tabular}{|c|c|c|c|c|}
\hline $\mathrm{Ze}_{\mathrm{Ti}}$ & $\alpha_{\mathrm{e}}$ & $\beta_{\mathrm{e}}$ & $\mathrm{A}_{\mathrm{e}}$ & $\mathrm{F}_{\mathrm{e}}$ \\
\hline 4.9 & 2.3 & 0.6 & 1.0 & 0.4 \\
\hline $\mathrm{Zp}_{\mathrm{p} \mathrm{Ti}}$ & $\alpha_{\mathrm{ep}}$ & $\beta_{\mathrm{ep}}$ & $\mathrm{A}_{\text {ep }}$ & $\mathrm{F}_{\text {ep }}$ \\
\hline 0.03 & 0.16 & 2.0 & 0.1 & 0.7 \\
\hline
\end{tabular}

The number of coefficient $\mathrm{N}$ in equation (10) is determined by minimization of chi square of fitting goodness of pair correlation function as shown in Fig. 1b. Minimal chi square is corresponding to $\mathrm{N}=70$ with the value of chi square $\chi^{2}=0.469583$. After fitted analytical form of pair correlation function of electron - positron $\mathrm{g}\left(\mathrm{r}, \rho^{-}, \rho^{+}\right)$is constructed, the enhancement factor is calculated with $\mathrm{g}\left(\mathrm{r}=0, \rho^{-}, \rho^{+}\right)=12.0676$ as shown in Fig. 1c. By applying the formular (9), annihilation rate is estimated by overlap of electron and positron densities, which is determined from Monte - Carlo simulation, and the enhancement factor $\Gamma=(2.25 \pm 0.03) \times 10^{9}\left(\mathrm{~s}^{-}\right.$ $\left.{ }^{1}\right)$. The positron lifetime is inverse of the annihilation rate $\imath=(444 \pm 6)$ ps. 

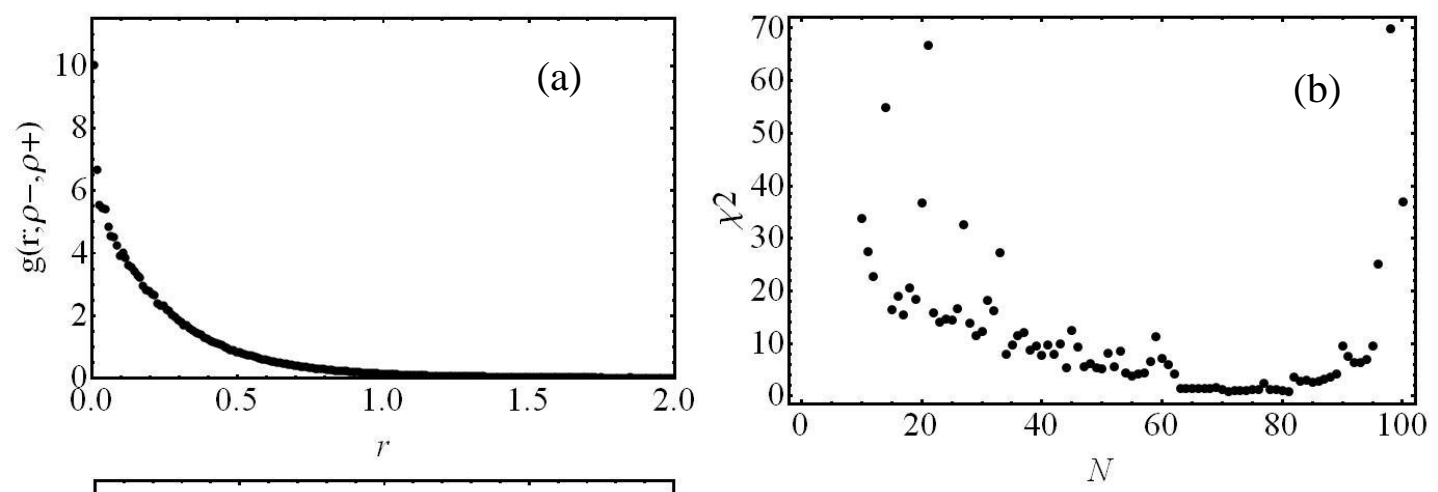

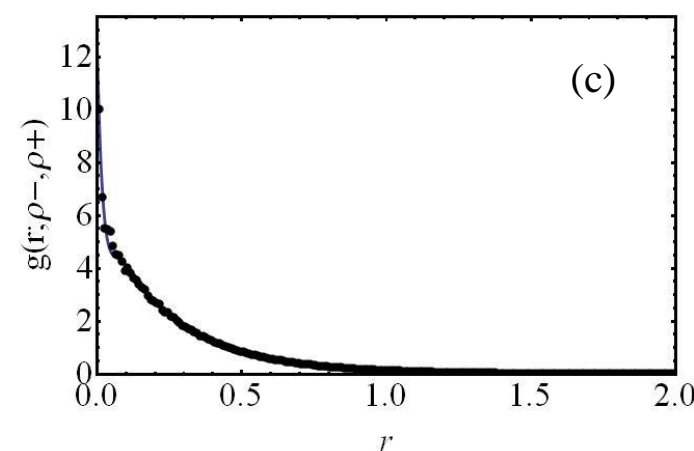

The positron lifetime $\mathrm{l}=444 \mathrm{ps}$ in single atomic titantium is greater than the result in titanium of bulk structure [1] which is 146ps. This deviation can be explained by the electron density of single titanium which is smaller than the electron density in titanium bulk.

The results of the enhancement factor and positron lifetime in zinc

Table 2. The values of optimized parameters of electron and positron wave function of zinc.

\begin{tabular}{|c|c|c|c|c|}
\hline $\mathrm{Ze}_{\mathrm{Zn}}$ & $\alpha_{\mathrm{e}}$ & $\beta_{\mathrm{e}}$ & $\mathrm{A}_{\mathrm{e}}$ & $\mathrm{F}_{\mathrm{e}}$ \\
\hline 3.7 & 4.3 & 0.5 & 1.0 & 0.9 \\
\hline $\mathrm{ZpZn}$ & $\alpha_{\mathrm{ep}}$ & $\beta_{\mathrm{ep}}$ & $\mathrm{A}_{\mathrm{ep}}$ & $\mathrm{F}_{\mathrm{ep}}$ \\
\hline 0.28 & 8.2 & 23.3 & 18.2 & 8.5 \\
\hline
\end{tabular}

The enhancement factor and positron lifetime in single atomic zinc are determined by using the
Fig. 1. The electron - positron pair distribution obtained by Monte - Carlo calculation with the optimized wave function of electron and positron in titanium (a); The chi square of goodness of fit in the term of the number of Chebyshev coefficients (b); The graph of fitted pair correlation fucntion of electron - positron in titanium. The black dot is Monte - Carlo simulation and the solid line is its fitting curve (c).

parameters in Table 2. The distribution of electron - positron pair correlation in zinc is obtained from Monte-Carlo's simulation as shown in Fig. 2a. The number of coefficient $\mathrm{N}$ in this case is identified by minimization of chi square coresponding to $\mathrm{N}=33$ with the value of chi square $\chi^{2}=8.08768 \times 10-6$ as shown in Fig. $2 \mathrm{~b}$. The enhancement factor in zinc is obtained by extrapolation of pair correlation function at $\mathrm{r}=$ 0 with $\mathrm{g}(\mathrm{r}=0, \rho-, \rho+)=20.0604$ as shown in Fig. 2c. The annihilation rate and lifetime of positron, which are determined from this enhancement factor and the overlap of electron and positron densities, are $\Gamma=2.181 \pm$ $0.005) \times 109 \quad(\mathrm{~s}-1)$ and $\mathrm{l}=(459 \pm 6) \mathrm{ps}$ respectively. 

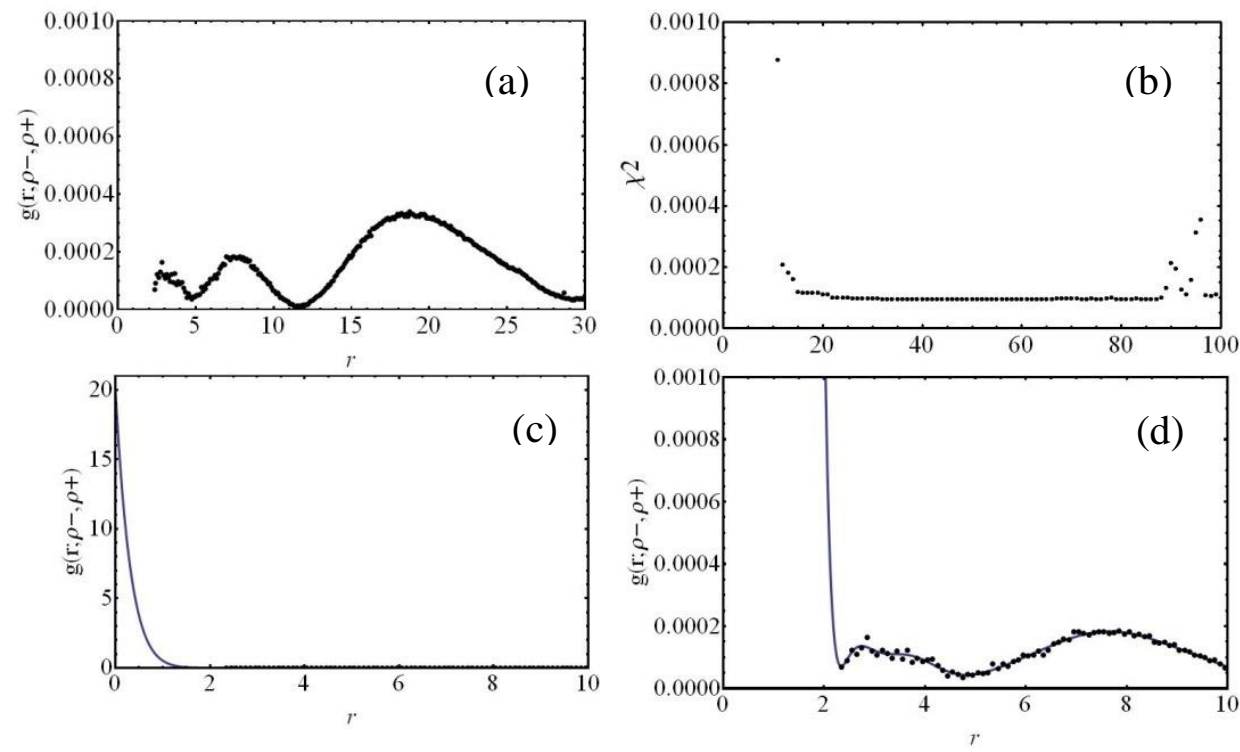

Fig. 2. The electron - positron pair distribution obtained by Monte - Carlo calculation with the optimized wavefunction of electron and positron in zinc (a); The chi square of goodness of fit in the term of the number of Chebyshev coefficients (b); The graph of fitted pair correlation function of electron - positron in zinc (c, d). The black dot is Monte - Carlo simulation and the solid line is its fitting curve.

The positron lifetime $\mathrm{l}=459 \mathrm{ps}$ in single atomic zinc is also greater than the results in zinc of bulk structure [1, 12-13] which are 148 ps, 198 ps and 266 ps respectively. In similarly to the titanium, this deviation can be also explained by the electron density of single zinc which is smaller than the electron density in zinc bulk.

\section{The results of the enhancement factor and} positron lifetime in zirconium

Table 3. The values of optimized parameters of electron and positron wave function of zirconium

\begin{tabular}{|c|c|c|c|c|}
\hline $\mathrm{ZeZr}$ & $\alpha_{\mathrm{e}}$ & $\beta_{\mathrm{e}}$ & $\mathrm{A}_{\mathrm{e}}$ & $\mathrm{F}_{\mathrm{e}}$ \\
\hline 5.9 & 9.0 & 0.5 & 0.5 & 0.3 \\
\hline $\mathrm{Zpzr}$ & $\alpha_{\text {ep }}$ & $\beta_{\mathrm{ep}}$ & $\mathrm{A}_{\mathrm{ep}}$ & $\mathrm{F}_{\mathrm{ep}}$ \\
\hline 0.03 & 0.1 & 1.7 & 1.5 & 7.6 \\
\hline
\end{tabular}

The enhancement factor and positron lifetime in single atomic zinc are determined by using the parameters in Table 3. The distribution of electron - positron pair correlation is obtained from Monte - Carlo's simulation as shown in Fig. 3a. The number of coefficient $\mathrm{N}$ is determined by minimization of chi square of fitting goodness of pair correlation function as shown in Fig. 3b. Minimal chi square is corresponding to $\mathrm{N}=17$ with the value of chi square $\chi 2=0.00101502$. By this pair correlation function, the enhancement factor is calculated with $\mathrm{g}\left(\mathrm{r}=0, \rho^{-}, \rho+\right)=4.40244$ as shonw in the Fig. 3c. The results of annihilation rate and life time of positron are $\Gamma=$ $(2.060 \pm 0.007) \times 109(\mathrm{~s}-1)$ and $\mathrm{\imath}=(485 \pm 2) \mathrm{ps}$ respectively.

\section{Trang 48}



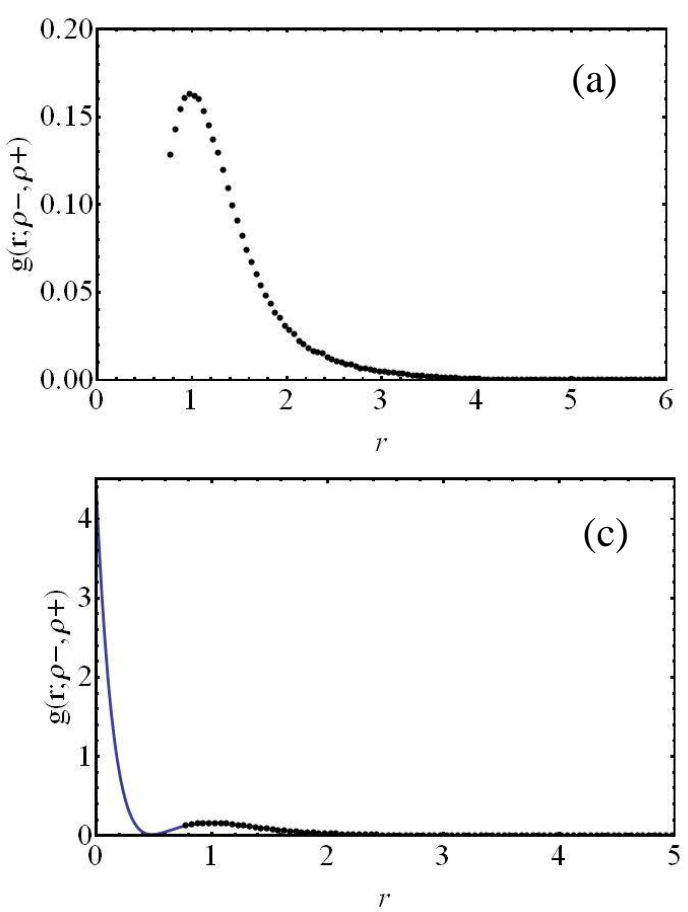

The positron lifetime $\mathrm{l}=485 \mathrm{ps}$ in single atomic zirconium is also greater than the result in zirconium of bulk structure [1] which is $172 \mathrm{ps}$. In similarly to the titanium and zinc, this deviation can be also explained by the electron density of single zirconium which is smaller than the electron density in zirconium bulk.

\section{CONCLUSION}

On the basic of the principle of linear superposition, Kohn - Sham approximation, the Slater-type orbital, the modification of Jastrow and VQMC method, we have managed to derive a theoretical model to determine the positron lifetime in some element - specific structure of materials.

In this scenario of pure theoretical calculations basing on real space electron and positron distributions, it is applied to estimate the value of the positron lifetime in single atoms

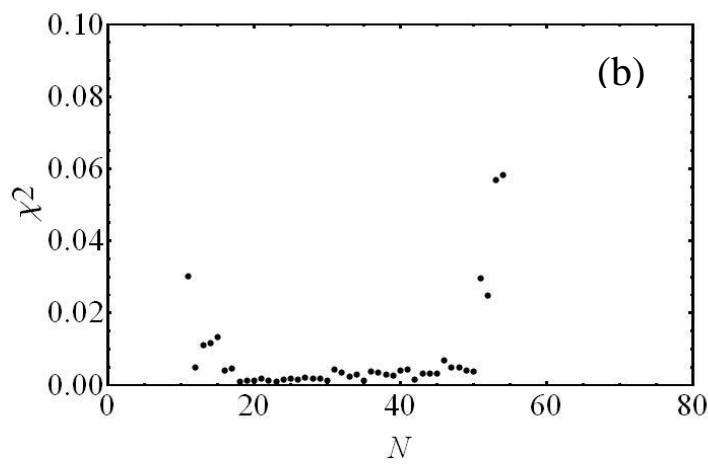

Fig. 3. The electron - positron pair distribution obtained by Monte - Carlo calculation with the optimized wavefunction of electron and positron in zirconium (a); The chi square of goodness of fit in the term of the number of Chebyshev coefficients (b); The graph of fitted pair correlation fucntion of electron - positron in zirconium (c). The black dot is Monte - Carlo simulation and the solid line is its fitting curve.

such as titanium, zinc and zirconium. The calculated results showed that the lifetimes of positron in single atoms of titanium, zinc and zirconium are greater than one in bulk structure of these atoms. This underestimation comes from the model of single atom whose valance electron densities is smaller than this one in bulk structure. So the positron lifetime in single atom will be greater than one in bulk and these theoretical results of this model are reasonable. These results pointed out that the positron lifetime will be increased by increment of atomic number because the coulomb interaction of nuclear charge and positron will become greater if atomic number increases and push positron more apart from valance electron. Therefore the positron has a chance to live more in heavier single atom. 


\section{Áp dụng xấp xỉ hàm sóng slater tính tốc độ hủy positron trong đơn nguyên tử}

- Trịnh Hoa Lăng

- Châu Văn Tạo

- Le Hoang Chien

- Huỳnh Thị Yến Hồng

- Huỳnh Ngọc Trâm

Trường Đại học Khoa học Tự nhiên, ĐHQG-HCM

\section{TÓM TÁT}

Thời gian sống và hệ số tăng cường hủy positron được tính bởi mô hình xấp xỉ liên kết chặt của hệ positron - vật chất. Trong đó hệ số tăng cường hủy được xác định từ hàm tương quan cặp. Hàm này được xác định từ những hàm sóng đơn hạt electron và positron đã được hiệu chỉnh phần hàm sóng tương quan electron - electron và electron positron. Động năng của electron và positron được xác định từ xấp xỉ Born - Oppenheimer

\section{REFERENCES}

[1]. M.J. Puska, Ab - initio calculation of positron annihilation rates in solids, J. Phys.: Condens. Matter, 3, 3455 - 3469 (1991).

[2]. G.F. Gribakin, C.M.R. Lee, Application of the zero-range potential model to positron annihilation on molecules, Nuclear Instruments and Methods in Physics Research B, 247, 31-37 (2006).

[3]. E. Boronski, R.M. Nieminen, Electron positron density - functional theory, Phys. Rev B, 34, 3820-3831 (1985).

[4]. W. Kohn, L.J. Sham, Self-consistent equations including exchange and correlation effects, Phys. Rev., 140, A1133-A1138 (1965). và xấp xỉ Kohn - Sham của phương trình đơn hạt. Sử dụng các hàm sóng đơn hạt của electron và positron theo xấp xỉ Slater cho các phương trình đơn hạt Kohn - Sham và áp dụng cho các electron hóa trị của các đơn nguyên tử và positron, các kết quả thời gian sống, tốc độ hủy và hệ số tăng hủy positron trong các đơn nguyên tử Ti, $\mathrm{Zn}$ và $\mathrm{Zr}$ được xác định.

[5]. H. Yukawa, On the Interaction of Elementary Particles, Progress of Theoretical Physics Supplement, 1, pp. 24-45 (1955).

[6]. V. Magnasco, Methods of Molecular Quantum Mechanics, University of Genoa, Genoa, Italy (2009).

[7]. S. Daiuk, M. Sob, A. Rubaszek, Theoretical calculations of positron annihilation with rare gas core electrons in simple and transition metals, Phys. Rev B, 22, 4, 2578-2593 (1991)

[8]. M. Hjorth, Jensen, Computational Physics, University of Oslo (2009).

[9]. M.J. Puska, R.M. Nieminen, Defect spectroscopy with positron: a general

Trang 50 
calculation method, J. Phys. F: Met. Phys, 43, 333-346 (1983).

[10].N.W. Ashcroft, N.D. Mermin, Solid State Physics, Holt-Saunders (1976).

[11].A. Gil, J. Segura, N. Temme, Numerical Methods for Special Functions, Society for Industrial Mathematics, 1 edition (2007).
[12].F. Plazaola, A.P. Seitsonen, M.J. Puska, Positron annihilation in II - V compound semiconductors: theory, J. Phys.: Condens. Matter, 6, 8809 - 8827 (1994).

[13].E. Boronski, R.M. Nieminen, Electron positron density - functional theory, Physical Review B, 34, 6, 3820 - 3831 (1986). 